\title{
Level of independence, functional capacity and respiratory muscle strength of patients with mucopolysaccharidosis type VI in the Northeast of Brazil
}

Bárbara Bernardo Rinaldo da Silva Figueirêdo 1

Paulo André Freire Magalhães 2

Livia Barbosa de Andrade 3

Patrícia Bezerra 4

Maria do Carmo Menezes Bezerra Duarte 5

1-5 Programa de Pós-graduação em Saúde Materno Infantil. Instituto de Medicina Integral Prof. Fernando Figueira. Rua dos Coelhos, 300. Boa Vista. Recife, PE, Brasil. CEP: 50.070-550. E-mail: barbara_bernardo@hotmail.com

\begin{abstract}
Objectives: to assess the functional independence, functional capacity and respiratory muscle strength (RMS) in individuals with mucopolysaccharidosis (MPS) type VI.

Methods: in this cross-sectional study, the Pediatric Evaluation of Disability Inventory (PEDI) and the Functional Independence Measure (FIM) scales were used to assess the functional independence. The functional capacity and the RMS were assessed by a 6-minute walk test (6MWT) and Manovacuometry, respectively. The associations between RMS and 6MWD were analyzed by using the adjusted simple linear regression models. And besides, the T-test was used to compare the differences among the groups.

Results: twenty-four patients with MPS VI were included, the median age was 8 (ranged from 2-38 years old). The PEDI presented a functional performance below the expectations at the self-care and mobility domains of $33.3 \%$ when compared to groups of Brazilian children at the same age assessed with typical development. The decreased social function was found in only one single case. According to the FIM, of the 12 patients evaluated (age $>7.5$ years), $58.3 \%$ were classified as modified dependence and $41.7 \%$ as modified independence. The mean distance in the 6MWT was significantly lower than predicted, $263 \mathrm{~m}$ in G1 (children, $n=7$ ) and $336 \mathrm{~m}$ in $G 2$ (adolescents and adults, $n=6$ ), $p<0.001$. The RMS was also lower than predicted in both groups, except for the RMS in G1.

Conclusions: the functional capacity and the RMS were significantly reduced in individuals with MPS VI, with a decreased functional independence in one-third of the patients. However, a multidisciplinary follow-up in this population is essential to prevent, diagnose and treat early complications.
\end{abstract}

Key words Physiotherapy, Mucopolysaccharidosis VI, Exercise tolerance, Respiratory muscles 


\section{Introduction}

Mucopolysaccharidosis type VI (MPS VI) or Maroteaux-Lamy syndrome (OMIM ID: 253200) is caused by a deficiency of the Arylsulfatase B enzyme. ${ }^{1}$ It is considered one of the rarest types in the world. While 1,100 individuals are estimated to have MPS VI, only $10 \%$ have been evaluated by a biochemical and genetic perspective. ${ }^{2}$ In Brazil and Portugal, the incidence of MPS VI is twice as much than those found in Australia and in the Netherlands. ${ }^{3,4}$ The MPS VI is one of the most commonly diagnosed types in Brazil, 1,5,6 with an estimated incidence of 0.083 per 100,000 inhabitants. Furthermore, it is more common in the Northeast of the country. ${ }^{2}$

The first signs of the disease appear at childhood, 6 including growth retardation, flexion contractures in the whole body, progressive joint stiffness, cardiovascular alterations and obstructive sleep apnea syndrome. 1,7 These abnormalities may result in a significant loss of the functions. ${ }^{8}$ Respiratory and cardiac symptoms reflect on these patients' intolerance to exercise, which is reported as a strong limiting factor in the daily life activities (DLA) and directly affecting the functional capacity. This also occurs due to the deposits of glycosaminoglycans (GAG) in the mouth, nose, throat and tracheobronchial cartilage. 9 Due to the range of alterations presented, the individuals die in their second or third decade of life. 1

There are no alterations related to cognitive function in patients with MPS VI and specific therapy consists of replacing the deficient enzyme with the objective to delay the progression of the disease.7,10 Therefore, early diagnosis and the initiation of enzyme replacement therapy (ERT) associated to multidisciplinary care are essential factors to improve these patients' quality of life. ${ }^{11}$

Studies addressing the assessment tools to determine functional independence highlight the Pediatric Evaluation of Disability Inventory (PEDI), used for measuring functionality in children 12 and the Functional Independence Measure (FIM) for older children and adults. ${ }^{13,14}$ To evaluate the functional capacity, studies have focused on the 6-minute walk test (6MWT) ${ }^{15}$ which has been used to assess the effect of the ERT on the functional capacity of individuals with MPS.7,16

Limitations in the musculoskeletal tissue also affect the respiratory muscles, harming the mechanic of the respiratory system in patients with MPS. $5,10,11$ The analysis on respiratory muscle strength is used as a predictor of morbidity and mortality in various populations, ${ }^{17,18}$ however, a medical literature review performed on Pubmed, SciELO and LILACS databases revealed few studies on patients with MPS. 19

The objective of this present study was to assess the level of the functional independence, the functional capacity and the respiratory muscle strength of patients with MPS VI.

\section{Methods}

A cross-sectional study was conducted after the approval by the Ethic Committee in Human Beings Research at Instituto de Medicine Integral Prof. Fernando Figueira (IMIP) under the document number; 3812-13. All the adult patients with MPS VI or, in the case of children and adolescents, their legal guardians signed an informed consent form and/or informed assent form prior to be admitted in the study.

Male and female individuals of 2 to 38 years of age with MPS VI were included in the study. A flow chart with the inclusion of the participants in the study is shown in Figure 1. Only patients over six years of age were invited to participate in the 6MWT and in the manometry tests.

Initially, the demographic, anthropometric and clinical data were registered on a standardized form containing the variables of interest to the study. Physical activity level was assessed by using the Habitual Level of Physical Activity (HLPA) questionnaire and classified as: sedentary lifestyle, regular physical activity (up to 2 hours/week) or organized/competitive sport/physical activity (more than 2 hours/week). 20

Specific questionnaires were applied to assess the functional independence. The Pediatric Evaluation of Disability Inventory (PEDI) ${ }^{12}$ was used in case of children aged two to seven years and 6 months. This consists of a structured questionnaire divided into three areas: social functions, self-care and mobility. Each item is assessed and the child receives a score of " 1 " if he/she is able to perform a task or " 0 " if he/she is unable to. These data were added and transformed from total crude scores to standardized (normative) scores that provided information on the expected performance of Brazilian children in the same age group with normal development. The scores were then classified into three levels: Level 0: 0-29 (development considered delayed or performance below than expected); Level 1: 30-70 (considered within the normal limits) and Level 2: $>70$ (performance significantly above that expected). 12 


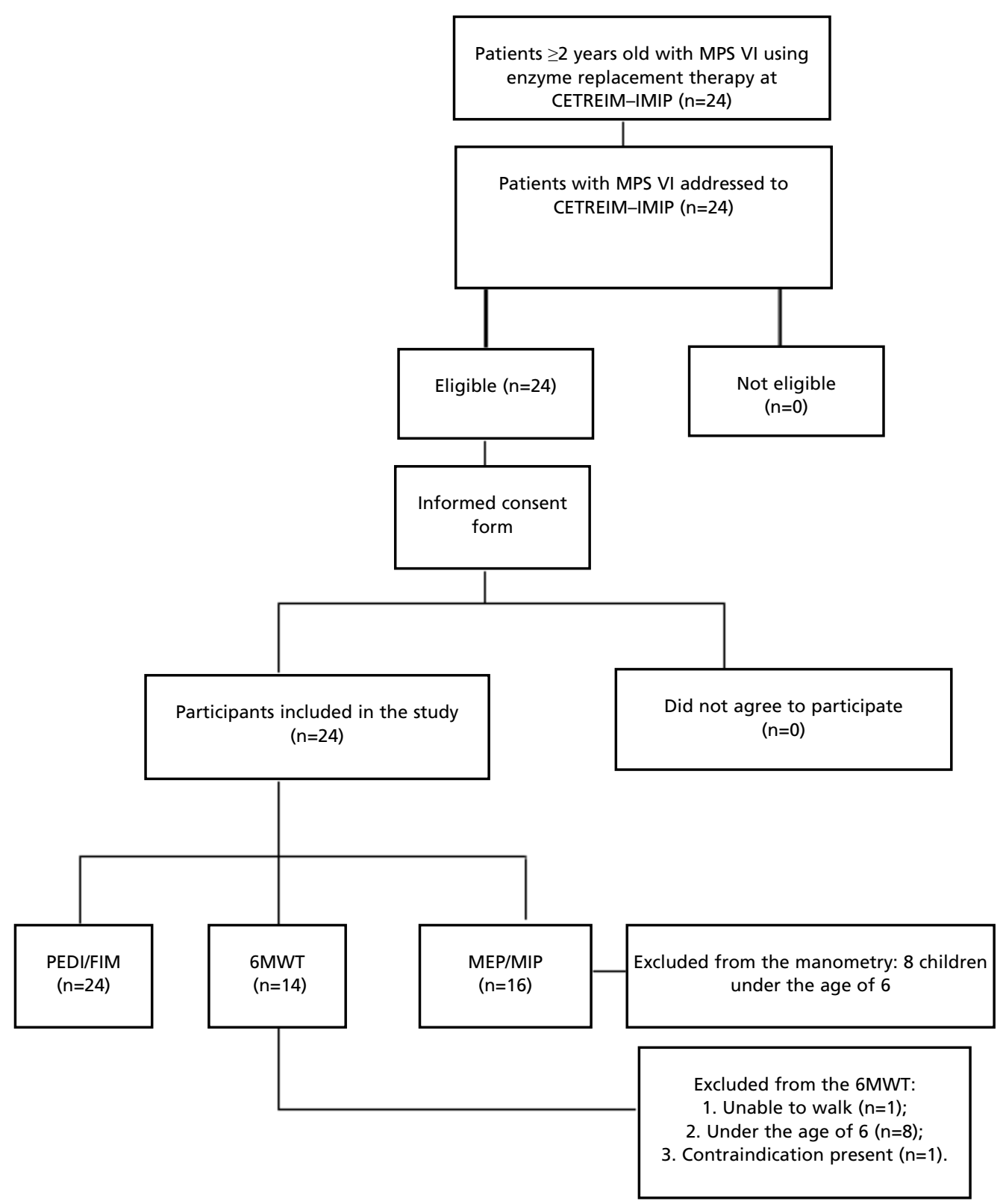

MPS VI: Mucopolysaccharidosis type VI; CETREIM-IMIP: A Treatment Center for Inborn Errors of the Metabolism at the Instituto de Medicina Integral Prof. Fernando Figueira; PEDI: Pediatric Evaluation of Disability Inventory; FIM: Functional Independence Measure; 6MWT: 6-minute walk test; MEP: maximal expiratory pressure; MIP: maximal inspiratory pressure.

The FIM was used to evaluate patients over 7 years and 6 months. This instrument assesses 18 items with scores that range from 1 to 7 , classifying the individuals according to their level of independence in performing the task. The items are grouped into six domains: self-care, sphincter 
control, transference, locomotion, communication and social cognition. Each domain was analyzed based on the sum of the relevant categories and lower scores indicated a greater degree of dependence. The overall score was obtained by adding the points in each of the FIM domains, reaching a total score that ranged from a minimum of 18 to a maximum of 126 points.

Dependence levels were then classified by subscores, with $\leq 18$ points reflecting complete dependence (full assistance required), 19-60 points: modified dependence/ moderate assistance (assistance required for up to $50 \%$ of the tasks), 61 103 points: modified dependence/ minimal assistance (assistance required for up to $25 \%$ of the tasks) and 104-126 (modified independence). 13 The PEDI and FIM were applied to the adults or caregivers / primary caregiver of the children and adolescents, personally by an only trained researcher.

The sub-maximal functional capacity was assessed on the patients over six years of age by using the 6MWT as standardized by the American Thoracic Society (ATS) 2002.15 Based on the reference values suggested by Priesnitz et al. 21 for healthy Brazilian children aged 6-12 years old. The predicted distance of the six-minute walk (6MWT) test was calculated. The equation established by Gibbons et al.22 was used to calculate the predicted distance for the individuals over 20 years old.

The maximum respiratory pressure was assessed by using an analogic manometer (Comercial Médica ${ }^{\circledR}$, São Paulo, Brazil). The test was performed in patients over six years old and seated in a comfortable position. The maximum inspiratory pressure (MIP) was measured by using an inspiratory maneuver starting with the functional residual capacity, while the maximum expiratory pressure (MEP) was measured beginning with the total lung capacity for a forceful exhalation by using a nose clip to disallow the flow of air through the nose. For both measurements, three maneuvers were performed, which the best of the three was selected according to the technique established in the study conducted by Wilson et al., 23 the MIP $(40+(0,57 \mathrm{x}$ weight $)$ and the MEP $(24+(4,8 \times$ age $)$. For the individuals over 18 years old, the values were established according to the predictive equations of Neder et al.,24 as for MIP in women (0,49 (age) + $110,4)$ and for men $(0,80$ (age) $+155,3)$, for MEP in women: 0,61 (age) + 115.6, and for men: 0,81 (age) $+165,3$. A height variable did not take part of the predictive equations.

In the descriptive data analysis, the frequency distribution tables were constructed and the central tendency and dispersion were calculated measurements. The Student's t-test was used in the inferential analysis to compare the means between the two groups. The associations among the MIP, MEP and the 6MWD were analyzed following the simple linear regression adjustments models considering the $6 \mathrm{MWD}$ as the response variable. The significance level was defined as $5 \%$ in all the tests. The statistical analysis was performed by using the Stata 12.1 SE software.

\section{Results}

Twenty-four patients with MPS VI were included in the study. The median age was 8 years old (ranged from 2-38 years old). In relation to their nutritional status, $12.5 \%$ had a body mass index (BMI) indicating underweight, while $25 \%$ presented to be overweight and $8.3 \%$ were obese. The most common phase of development was pre-pubertal $(62.5 \%)$. The median height was 1.06 meters, considered as low height (Table 1).

As for per capita income, $79 \%$ received between one and two minimum salaries. The median number of years of the patient's schooling was four, varying from $0-18$ years, while the median age of the first diagnosis was at the age of two years old, varying from 2 months to 16 years old. Sedentary lifestyle was present in $70.8 \%$ of the cases and three-quarters of the patients did not have frequent weekly physiotherapy sessions. The duration of ERT varied from 1 to 79 months, with a median of 41.5 months (Interquartile Range [IQR]: 19.5-58.5 months) (Table 1). In relation to the ERT duration, 7 patients were doing ERT between 1 and 24 months, 8 patients were doing between 24 and 48 months, and 9 patients were doing ERT for over 48 months.

Due to clinical manifestations related to MPS VI, it is necessary multi, inter and transdisciplinary follow-ups to prevent and diagnose early complications, which should not only be treated with ERT. A serious and concerning discovery is the absence of physiotherapeutic treatment, which is essencial to attenuate the progression of the disease and to provide better functionality.

The PEDI was applied on 12 children aged 2-7 years and 5 months old, and their normative scores are shown in Table 2. The median normative scores on self-care and mobility were 32 and 33 , respectively, indicating that $33.4 \%$ of the patients had a delay in the development or the performance was below the expectations for healthy Brazilian children in the same age group. In the area of social 
Characteristics of the 24 patients with mucopolysaccharidosis type VI being followed up at a Treatment Center for Inborn Errors of the Metabolism in the Northeast of Brazil, 2014.

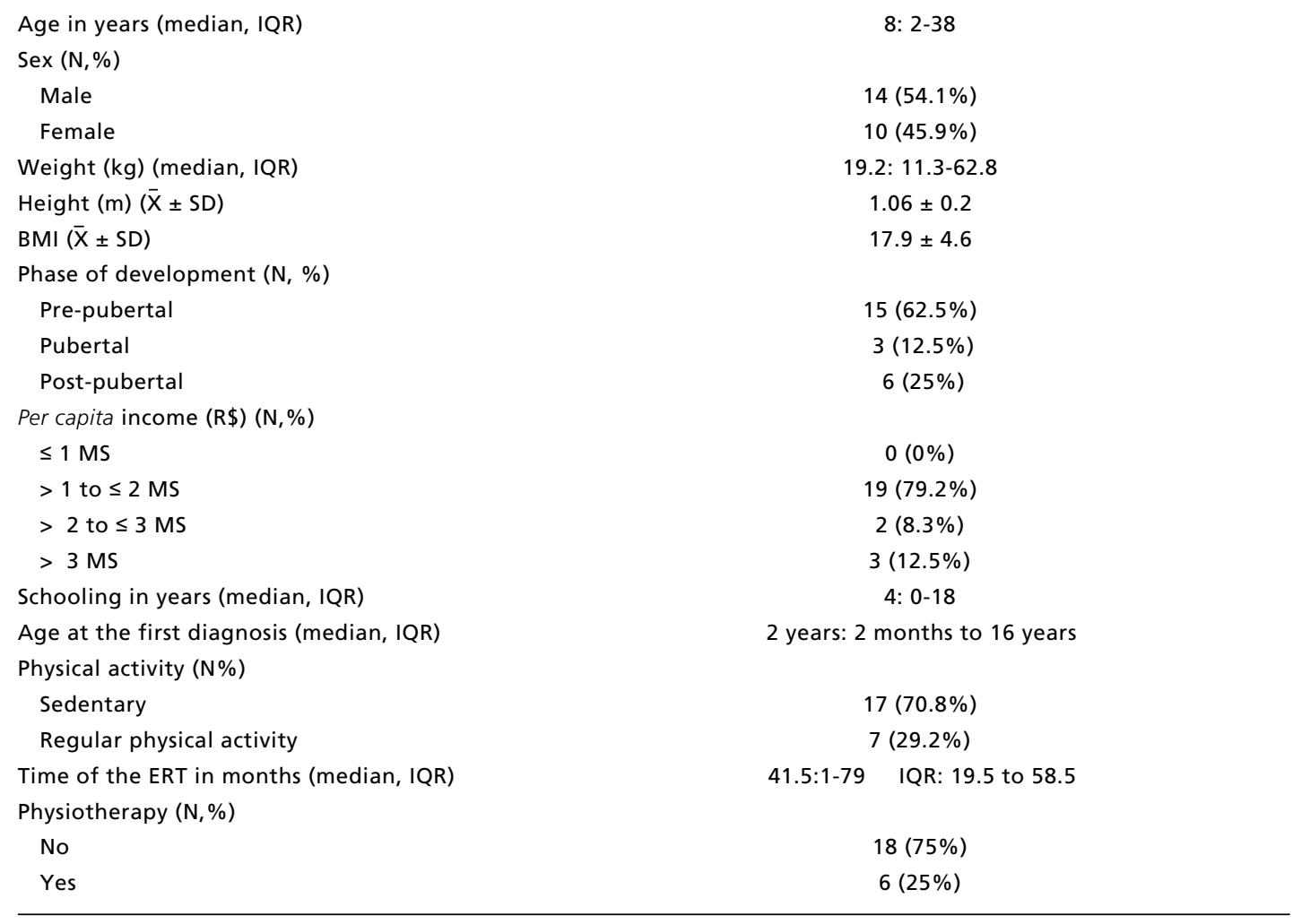

Value expressed as med= Medians and in standard deviations means (SD); $N \%$ = number of patients.,percentage $\mathrm{BMI}=$ Body Mass Index, MS= minimum salary at the time (2014) R $\$ 724.00 ; \mathrm{IQR}=$ Interquartile range $25-75 \%$.

function, the mean normative score was 49 , indicating that $91.7 \%$ of the patients were within the normal range for healthy Brazilian children of the same age group (Table 2).

Twenty four patients assessed, twenty of them had better mobility, the duration of ERT was significantly higher in patients $(n=4)$ with delayed development or those who performed below the expectations (37 months $\mathrm{x} \quad 16.5$ months, respectively; $p=0.042$ ). No association was found between the duration of ERT and the items in the self-care domain.

In the 12 patients assessed, the functional independence used the FIM and obtained a median score equal to 101.5. The modified dependence (assistance required in up to $25 \%$ of the tasks) was observed at $58.3 \%$ and the modified independence was at $41.7 \%$ (Table 2). The duration of ERT was significantly higher in those that presented having modified dependence (assistance required in up to $25 \%$ of the tasks) compared to those patients that presented modified independence $(p=0.005)$. The five patients that were found to be in the modified independence category, three of them presented an attenuated form of the disease.

The mean values found in the 16 patients who performed the respiratory muscle strength (MIP and MEP) assessment are shown in Table 3. Eight patients did not perform the assessment because they were under the age of 6 .

The mean values found in the 14 patients who performed the 6MWT were lower than the predicted values for the same age group (Table 3). When the mean 6MWT was compared to the overall FIM scores, there was no statistically significant difference found among the patients that presented a modified independence and those that presented a modified dependence (assistance in $u$ to $25 \%$ of the tasks $)(p=0.264)$.

Immediately after the 6MWT, the mean Borg score was $6.3 \pm 1.5$ in the group of adolescents and the adults and $6.5 \pm 3.7$ in the group of 
children, although, there was no need to interrupt the test with any of the assessed patients. There was no significant correlation observed between the duration of ERT and the functional capacity, $(\mathrm{r}=$ $0.438),(p=0.117)$ The linear regression showed a positive association $(p=0.001)$ between the MIP and 6MWD variables indicating that the higher the MIP, the longer is the distance performed by the patient with an estimated increase of 1.7 meters in 6MWD for each increase of $1 \mathrm{cmH}_{2} \mathrm{O}$ of the MIP. For the
MEP, no statistically significant association was found in the 6MWD. When comparing the distance mean performed by the group of patients $(n=6)$ who had physiotherapy sessions once a week (305.2 \pm 55.5 meters) with those $(\mathrm{n}=8)$ who did not do (295.9 \pm 95.6 meters). However, it was observed that the individuals who had physiotherapy were able to walk 9.3 meters more, but without statistical significance $(p=0.823)$.

Table 2

Level of functional independence in 24 patients with mucopolysaccharidosis type VI being followed up at a Treatment Center for Inborn Errors of the Metabolism in the Northeast of Brazil, 2014.

\begin{tabular}{|c|c|}
\hline Variables & Med, IQR \\
\hline \multicolumn{2}{|l|}{ Functional independence $n=24$} \\
\hline \multicolumn{2}{|l|}{ PEDI $n=12$} \\
\hline Self-care (median, IQR) & 32: $19.5-45.6$ \\
\hline Delayed development or performance below the expectations* & $4(33.4 \%)$ \\
\hline Within normal standards * & $8(66.6 \%)$ \\
\hline Performance significantly above the expectations * & $0(0 \%)$ \\
\hline Mobility (median, IQR) & 33: $21-42$ \\
\hline Delayed development or performance below the expectations* & $4(33.4 \%)$ \\
\hline Within normal standards * & $8(66.6 \%)$ \\
\hline Performance significantly above the expectations * & $0(0 \%)$ \\
\hline Social function (median, IQR) & 49.2: (28.1-62) \\
\hline Delayed development or performance below the expectations* & $1(8.3 \%)$ \\
\hline Within normal standards * & $11(91.7 \%)$ \\
\hline Performance significantly above the expectations * & $0(0 \%)$ \\
\hline FIM $n=12$ (median, IQR) & 101.5: $65-115$ \\
\hline Complete dependence* & $0(0 \%)$ \\
\hline Modified dependence (up to $50 \%$ of the tasks)* & $0(0 \%)$ \\
\hline Modified dependence (up to $25 \%$ of the tasks)* & $7(58.3 \%)$ \\
\hline Modified independence* & $5(41.7 \%)$ \\
\hline
\end{tabular}

Values expressed Median (Med) and amplitude; *Numbers ( $n$ ) and percentages (\%); PEDI= Pediatric Evaluation of Disability Inventory; FIM= Functional Independence Measure; IQR= Interquartile range $25-75 \%$. 
Comparison of the mean obtained and the predicted respiratory muscle strength and the functional capacity of patients with mucopolysaccharidosis type VI attended at the Treatment Center for Inborn Errors of the Metabolism in the Northeast of Brazil, 2014.

\begin{tabular}{lcccc}
\hline Variables & Groups * & $\begin{array}{c}\text { Mean value } \\
\text { obtained }\end{array}$ & $\begin{array}{c}\text { Mean value } \\
\text { predicted }\end{array}$ & $p^{* *}$ \\
\hline & & & & \\
Respiratory muscle strength $(\bar{X} \pm S D) n=16$ & $1(n=10)$ & $-43.2 \pm-25.7$ & -58.2 & 0.097 \\
$\quad$ MIP & $2(n=6)$ & $-77 \pm-42.9$ & -152 & 0.008 \\
MEP & $1(n=10)$ & $59.6 \pm 20.4$ & 80.3 & 0.011 \\
& $2(n=6)$ & $83.7 \pm 33.9$ & 161 & 0.002 \\
Distance in meters $(\bar{X} \pm S D) n=14$ & & & & $<0.001$ \\
Distance walked & $1(n=7)$ & $263.1 \pm 69.6$ & 507 & $<0.001$ \\
\end{tabular}

Values expressed in means and standard devation (SD); *Group 1: children, Group 2: adolescents and adults; ** Student's t-test; MIP= maximal inspiratory pressure; MEP= maximal expiratory pressure.

\section{Discussion}

This present study shows that assessed patients with MPS type VI had significant reduction on the functional capacity and respiratory muscle strength and one-third of the patients had a delay in the functional independence level. The median age of the first diagnosis found was at the age of two years old. 14 patients $(58.3 \%)$ received diagnosis prior to complete three years of age. The data on Management Guidelines for Mucopolysaccharidosis VI 25 state that diagnosis in this population is generally performed between one and three years of age, this is the age in which the first signs of the disease appear. In Brazil, a study involving 113 patients with MPS, (29 with MPS VI) found that the mean age between the appearance of the first signs and symptoms of the disease and confirmation of its diagnosis was of 76 months and 52 months for those with MPS VI, considering the group as a whole in this study had a delay of 4.8 years between the beginning of the signs/symptoms and the diagnostic. 5

The delayed diagnosis in the present study was verified in only three patients (two 9 years old and one 16 years old), possibly due to the attenuated form of the MPS VI and/or by the difficulty of health professionals in identifying the disease.

The patients' height deficit found in the present study is in agreement with the Guidelines for Mucopolysaccharidosis VI25 in which describes this finding as being one of the first signs of MPS type VI, reporting a mean height of $95-150 \mathrm{~cm}$. The BMI mean was $17.9 \mathrm{~kg} / \mathrm{m}^{2}$, lower than those found in Matos et al.8 study by comparing the energy expenditure of walking of 19 individuals with MPS (type unspecified) and 19 individuals without MPS from the community (comparison group), where the observed BMI mean was $19.2 \mathrm{~kg} / \mathrm{m}^{2}$ in patients with the disease and verified that the patients with MPS were not significant different of the control individuals in relation to the BMI, although, there was a difference according to height and weight. On the other hand, Lin et al. 26 assessed 35 patients with MPS, six of whom had MPS VI, a BMI mean of 20.6 $\mathrm{kg} / \mathrm{m}^{2}$, a higher value found than in this present study.

In the functional independence assessment, there was a greater functional impairment in the areas of self-care and mobility by using both instruments: the PEDI and FIM. These findings could be explained due to the joint stiffness, (particularly in the knees, hips, wrists and elbows), 27 which are secondary to the metaphyseal involvement and the thickening and the fibrosis joint capsule. These osteoarticular manifestations are progressive and for this reason, they limit the movements. ${ }^{1}$

The joint stiffness in the shoulder, knee and elbow jeopardize considerably the activities such as brushing the teeth and hair, eating and walking 28 as in this present study in which the activities reported as being the most difficult were in the areas of selfcare and mobility (brushing the hair, buttoning and unbuttoning clothes, tying shoe laces, climbing up stairs and walking more than 30 meters).

A researched literature with the descriptors: 
evaluation, physical therapy specialty and mucopolysaccharidosis VI conducted between 20002014 found only one Brazilian study involving patients with MPS using the PEDI and the FIM.7 Twenty-one patients were included in that study, 4 with type VI and the others with type I, II, III or IV, mobility was assessed in that study and the joints studied presented limitations, especially in the MPS I,II and VI. 7

In the social function, $91.7 \%$ of the assessed children using the PEDI were within the normal range as expected for Brazilian children at the same age. This finding is justified by the fact that the MPS VI, as well as Morquio syndrome (MPS VI) have cognitive function and preserved intelligence. ${ }^{1}$ Only one child in the present study presented a considerable delay or performance below the expectation score. Mizuno et al. ${ }^{1}$ study describes the learning and behavioral alterations found in some of these patients that could be the result of a sleep disorder, leading to daily drowsiness and missing school days.

Regarding to the FIM, the social cognition, this present study verified that in half of the patients (6) were found to be dependent on parents/legal guardians. This fact may be explained because these patients are under the age of 18 (depend on caregivers) and have impaired visual acuity. A Brazilian study by Guarany et al.,7 involved 21 patients with various types of MPS (four with MPS VI) raised the possibility that patients' cognitive acquisition may be impaired due to their sensory deficit and physical limitations. The physical limitations interfere progressively in their learning and motor skills development, determining a reduction of the physical and functional capacity, affecting their wellbeing and leading to a reduction of their useful life. The preservation of functionality is a growing challenge in the treatment of patients with MPS, and the maintenance of functionality should be the main objective in the treatment of these patients. It should be emphasized that the FIM and the PEDI are instruments that assess the function in a quantitative form, they assess the burden of care required by an individual to perform a series of motor and cognitive tasks in their daily lives, 12,13 however, they do not assess the quality/form in which the activity is performed (motor coordination, involvement of muscle groups to perform the task, amplitude of movements, muscle strength and fine motor coordination).

In the mobility domain, we found the duration of ERT significantly greater in individuals with delayed development or a performance that was below the expectations. These findings differ from the results from a multicenter study conducted by Harmatz et al. ${ }^{29}$ who assessed ten patients with MPS VI and found that the duration of ERT was associated to a reduction in the urinary excretion of GAG, with the improvement in the respiratory function and in the functional capacity to walk and climb stairs, as well as in a perceived improvement in joint stiffness and pain, with an increase in the amplitude of movement. Nevertheless, another study involving 22 patients with MPS, 11 of whom had MPS VI, found that after one year of ERT motor function measured by the amplitude of movement showed little clinical improvement. 8

In a study in Brazil involving 28 patients with MPS VI who had no physiotherapy, 27 obtained a mean walking distance of 258.5 meters in the $6 \mathrm{MWT}$, a similar result was found in this study. In this present study, the difference between the walking distance by the patients was statistically lower than those predicted in the two studies. This may be explained due to the multi-systemic manifestations of the disease (bone deformities, joint disease, heart disease and respiratory, visual deficit and compression of the spinal cord). $.8,9$ The relation observed between the patients with longer duration of ERT and the shortest walking distance in the $6 \mathrm{MWT}^{\prime}$ can be explained by the natural progression of the disease, where the affected individuals are usually dependent of wheelchairs or bedridden due to several changes. 29

The macroglossia and mandibular alterations present in some types of MPS including in the MPS VI, may lead to an increased risk of progressive airway obstruction and sleep apnea syndrome. Respiratory problems are cited as the main reasons for hospitalizations. ${ }^{1,9}$

In the databases investigated, an abstract was found in which a significant reduction in respiratory muscle strength was detected using the manometry. ${ }^{19}$ In that study involving seven patients with MPS VI, the level of dyspnea during DLA was also assessed, resulting in a Borg indice of 7 , indicating intense dyspnea during daily activities. 19 In the MPS VI, pulmonary function and the ventilatory capacity can be directly affected by the short height and skeletal dysplasia 9,25 interfering in the MIP values. This fact may justify the lowest values found in our study. Despite the importance of this study, some limitations should be mentioned. First, the PEDI and the FIM questionnaires used to assess functionality are not specific for patients with MPS. Secondly, the questions were made directly to the patient with the agreement of their parents or 
legal guardian, and in some cases (in the case of the smaller children) directly to the parent or the legal guardian. Thirdly, the sample size was small. Nevertheless, to the best of our knowledge, this is the largest study conducted in Brazil with the aim of assessing functional capacity, functional independence level and respiratory muscle strength in patients with MPS VI.

In conclusion, this present study showed that functional capacity and respiratory muscle strength were significantly reduced in individuals with MPS VI, (maximal inspiratory pressure and maximal expiratory pressure) with the decreased functional

\section{References}

1. Mizuno CA, Figueiredo JB, Teza ITV. Aspectos clínicos da mucopolissacaridose tipo VI. Rev Soc Bras Clin Med. 2010; 8 (4): 356-61.

2. Costa-Motta FMM, Acosta AX, Abé-Sandes K. Genetic studies in a cluster of mucopolysaccharidosis type VI patients in Northeast Brazil. Mol Genet Metab. 2011; 104: 603-7.

3. Pinto R, Caseiro C, Lemos. Prevalence of lysosoma storage diseases in Portugal. European journal of human genetics: EJHG. 2004; 12: 87-92.

4. Coelho JC, Wajner M, Burin MG. Selective screening of 10,000 high-risk Brazilian patients for the detection of inborn errors of metabolism. Eur J Pediatr. 1997; 156: 650-

5. Vieira T, Schwartz I, Muñoz V. Mucopolysaccharidoses in Brazil: what happens from birth to biochemical diagnosis? Am J Med Genet. 2008; 146: 1741-7.

6. Azevedo ACMM, Schwartz I, Kalakun L. Clinical and biochemical study of 28 patients with mucopolysaccharidosis type VI. Clin Genet. 2004; 66 (3): 208-13.

7. Guarany NR, Schwartz IVD, Guarany FC. Functional capacity evaluation of patients with mucopolysaccharidosis. J Pediatr Rehab Med. 2012; 5: 37-46

8. Matos MA, Barreto R, Penha CE. Amplitude de movimentos em pacientes com mucopolissacaridose (MPS-I MPS-II E MPS-VI), após um ano de terapia de reposição enzimática. Rev Baiana Saúde Pública. 2010; 4 (1): 60-1.

9. Berger KI, Fagondes SC, Giugliani R. Respiratory and sleep disorders in mucopolysaccharidosis. J Inherit Metab Dis. 2013 ; 36: 201-10

10. Giugliani R, Federhen A, Rojas MVM. Terapia de reposição enzimática para as mucopolissacaridoses I , II e VI recomendações de um grupo de especialistas brasileiros. Rev Assoc Med Bras. 2010; 56: 257-77.

11. Solanki G, Alden TD, Burton BK. A multinational, multidisciplinary consensus for the diagnosis and management of spinal cord compression among patients with mucopolysaccharidosis VI. Mol Genet Metab. 2012; 107 (1-2): 15-24 independence in one third of the patients. In view of the multi-systemic involvement of patients with MPS VI, interdisciplinary follow-up is important in order to prevent, diagnose and treat complications early. Therefore, multidisciplinary assessment is essential in caring for this population, since the aim of the treatment and rehabilitation in general should be focused on the individual's functionality. The functional independence is strongly linked to the quality of life and it is the objective for the rehabilitation team to be responsible for the treatment of the patients with MPS VI.

12. Mancini MC. Inventário de Avaliação Pediátrica de Incapacidade (PEDI). In Manual da versão Brasileira adaptada (Pediatric Evaluation of Disability Inventory (PEDI). Editora UFMG. 2005; p.17-22.

13. Riberto M, Miyazaki MH, Jucá SSH. Validação da Versão Brasileira da Medida de Independência Funcional. Acta Fisiatrica. 2004; 11: 3-7.

14. Scarpa M, Almássy Z, Beck M. Mucopolysaccharidosis type II: European recommendations for the diagnosis and multidisciplinary management of a rare disease. Orphanet $\mathbf{J}$ Rare Dis. 2011; 6: 2-18

15. American Thoracic Society. Guidelines for the six-minute walk test. Am J Resp Crit Care Med. 2002; 166 (1) : 111-7.

16. Swiedler S, Beck M, Bajbouj M. Threshold effect of urinary glycosaminoglycans and the walk test as indicators of disease progression in a survey of subjects with Mucopolysaccharidosis VI (Maroteaux-Lamy syndrome). Am J Med Genet. 2005; 134A(2): 144-50.

17. Carvalho EM, Isem MRM, Lima PA. Força muscular e mortalidade na lista de espera de transplante de fígado. Rev Bras Fisioter. 2008; 12 (3): 235-40.

18. Naso FCD, Pereira JS, Dias AS. Correlações entre variáveis respiratórias e funcionais na insuficiência cardíaca. Rev Portug Pneumol. 2009; XV(5): 875-90

19. Silva APZB, Taques PIDPP, Silva EKR. Avaliação da força muscular respiratória e da expansibilidade torácica em crianças e adolescentes portadores de Mucopolissacaridose tipo VI. AssoBraFIR Ciência. 2012; 3(Supl.): 44 Disponível em: www.uel.br/revistas/uel/index.php/rebrafis/ article/download/13991/11807

20. Santuz P, Baraldi E, Filippone M. Exercise performance in children with asthma: is it different from that of healthy controls? Eur Respir J. 1997; 10 (6): 1254-60.

21. Priesnitz CV, Rodrigues GH, Stumpf CS. Reference values for the 6-min walk test in healthy children aged 6-12 years. Pediatric Pulmonol. 2009; 44 (12): 1174-9.

22. Gibbons WJ Fruchter N, Sloan S. Reference values for a multiple repetition 6-minute walk test in healthy adults older than 20 years. J Cardiopulm Rehabil. 2001; 21 (2): 87-93. 
23. Wilson SH, Cooke NT, Edwards RH. Predicted normal values for maximal respiratory pressures in caucasian adults and children. Thorax. 1984; 39 (7): 535-8.

24. Neder JA, Andreoni S, Castelo-Filho A. Reference values for lung function tests. I. Static volumes. Braz J Med Biol Res. 1999; 32: 703-17.

25. Giugliani R, Harmatz P, Wraith JE. Management guidelines for mucopolysaccharidosis VI. Pediatrics. 2007; 120 (2): 405-18.

26. Lin SP, Shih SC, Chuang CK. Characterization of pulmonary function impairments in patients with mucopolysaccharidoses-changes with age and treatment. Pediatric Pulmonol. 2013; 49 (3): 277-84.
27. Cardoso-Santos A, Azevedo ACMM, Fagondes S. Mucopolysaccharidosis type VI (Maroteaux-Lamy syndrome): assessment of joint mobility and grip and pinch strength. J Pediatr. 2008; 84 (2): 130-5.

28. Pereira JO, Gabriela M, Moreira DA. Mucopolissacaridose tipo VI: evolução natural, importância diagnóstica e terapêutica. Rev Neurocienc. 2011; 19 (2): 329-38.

29. Harmatz P, Ketteridge D, Giugliani R. Direct comparison of measures of endurance, mobility, and joint function during enzyme-replacement therapy of mucopolysaccharidosis VI (Maroteaux-Lamy syndrome): results after 48 weeks in a phase 2 open-label clinical study of recombinant human $\mathrm{N}$-acetylgalactosamine 4-sulfatase. Pediatrics. 2005; 115 (6): 681-9.

Received on October 16, 2017

Final version presented on January 19, 2018

Approved on January 28, 2018 